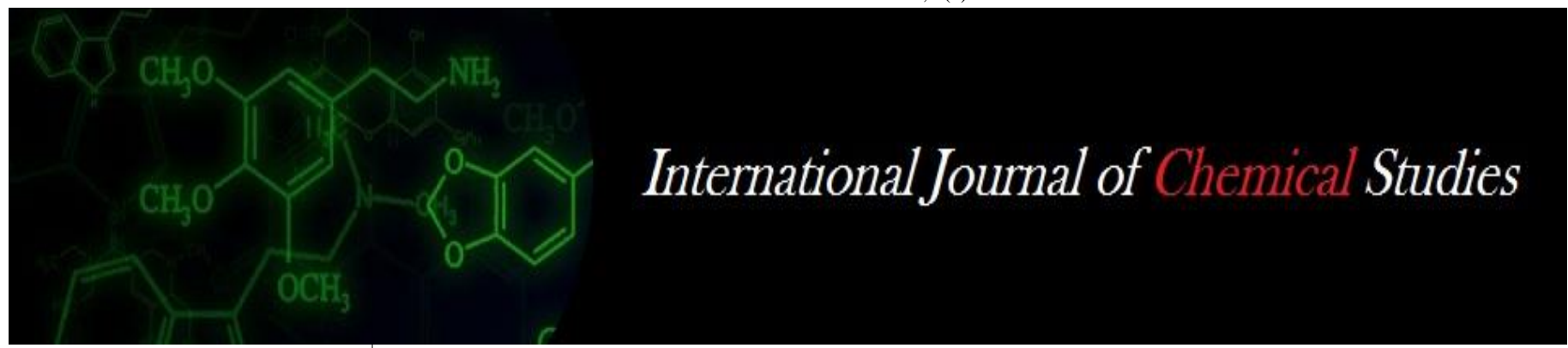

P-ISSN: 2349-8528

E-ISSN: 2321-4902

www.chemijournal.com

IJCS 2020; 8(6): 315-320

(C) 2020 IJCS

Received: 10-09-2020

Accepted: 12-10-2020

\section{GM Malve}

Ph. D. Scholar, Department of Horticulture, PGI, MPKV,

Rahuri, Maharashtra, India

\section{MN Bhalekar}

Senior Vegetable Breeder, AICRP (VC), Department of Horticulture, MPKV, Rahuri,

Maharashtra, India

\section{DB Kshirsagar}

Associate Professor, Department of Horticulture, PGI, MPKV, Rahuri, Maharashtra, India
Corresponding Author: GM Malve

Ph. D. Scholar, Department of Horticulture, PGI, MPKV, Rahuri, Maharashtra, India

\section{Studies on Heterosis in ridge gourd (Luffa acutangula (L.) Roxb.) in summer season}

\author{
GM Malve, MN Bhalekar and DB Kshirsagar
}

DOI: https://doi.org/10.22271/chemi.2020.v8.i6e.10786

\begin{abstract}
Heterosis study was under taken in $28 \mathrm{~F}_{1}$ hybrids of ridge gourd obtained from half diallel by using eight parental lines for fruit yield and its contributing characters during summer season. The complete sets of 36 genotypes were evaluated in a randomized block design (RBD) with two replications during summer season. For fifteen different characters the magnitude of heterosis noted in percentage increase or decrease over better parent, top parent and standard check. The cross combinations RHR RG-1 x RHR RG-8, RHR RG-2 x RHR RG-7, RHR RG-2 x RHR RG-8, RHR RG-5 x RHR RG-8, RHR RG-6 x RHR RG-7, RHR RG-6 x RHR RG-8, displayed the significant positive heterosis for most of the traits in summer season. As earliness is a desirable character in ridge gourd, the significantly negative heterosis is considered desirable for all maturity traits viz., length of internode, days to $50 \%$ flowering, length of pedicel and days required for first harvest.
\end{abstract}

Keywords: Luffa acutangula, heterosis, hybrid, diallel, heterobeltosis

\section{Introduction}

Ridge gourd (Luffa acutangula (L.) Roxb.) is one of the popular vegetable crop belonging to the family cucurbitaceae. Ridge gourd has good nutritive value, rich in carbohydrates and contains the minerals $\mathrm{Ca}$. P and Fe. It is also a good source of vitamin A, vitamin B and a fair source of ascorbic acid. Exploitation of hybrid vigour in recent years has led to remarkable yield advances in many crops. However, the possibility in exploitation of hybrid vigour in ridge gourd as a result of inter-varietal crossing was shown by Richharia (1952) ${ }^{[11]}$. Owing to existence of wide variability, monoecious nature, conspicuous and convenient flower and quite large number of seeds per fruit. Ridge gourd can serve as a good source for the manifestation of the heterosis and its commercial exploitation. Besides this, cucurbits are distinct group among the cross pollinated crops that they do not suffer much from inbreeding depression. Thus, heterosis breeding can prove as a useful tool in ridge gourd improvement. When two genetically differing individuals are crossed. The increased vigour obtained in the descendants is called hybrid and it is expressed as heterosis percentage increase over the parents. Heterosis breeding has been a recognized practical tool in providing the breeder a means for increasing the yield and other economic traits. The hybrid vigour or the superiority of the $F_{1}$ hybrids over parents may be manifested in terms of high productivity, uniformity, improved quality, built in resistance, environmental condition, earliness etc. However, it never happens that each hybridization is accompanied by the manifestation of hybrid vigour. Only certain pair of parents gives heterotic progeny. Therefore, for development of an effective heterosis breeding programme in ridge gourd one needs to elucidate the genetic variance nature and magnitude of quantitatively inherited traits and estimate parents in hybrid combinations.

\section{Material and methods}

The experiment was laid out during July, 2017 to May, 2018 at All India Coordinated Research Project on Vegetable crops, Department of Horticulture, Mahatma Phule Krishi Vidyapeeth, Rahuri in Randomized Block Design (RBD) with two replications. The experiment consists of eight parents (RHR RG-1, RHR RG-2, RHR RG-3, RHR RG-4, RHR RG-5, RHR RG-6, RHR RG-7 and RHR RG-8), twenty eight $F_{1}$ hybrids and one standard check; each entry was planted in rows of five vines each. A spacing of $1.5 \times 1.0 \mathrm{~m}$ was followed and the crop was raised as per the recommended package and practices. 
The all parents, hybrids and standard check were randomized completely among themselves and grown in continues block. Recorded parameters viz., length of vine $(\mathrm{cm})$, number of nodes per vine, length of internode $(\mathrm{cm})$, number of female flowers, days to $50 \%$ flowering, days required for first harvest, length of fruit $(\mathrm{cm})$, diameter of fruit $(\mathrm{cm})$, length of pedicel $(\mathrm{cm})$, number of ridges on fruit, weight of fruit $(\mathrm{g})$, number of fruits per vine, weight of fruits per vine $(\mathrm{kg})$, weight of fruits per plot $(\mathrm{kg})$, fruit yield $(\mathrm{q} / \mathrm{ha})$. The experimental data analysed by statistical method suggested by Panse and Sukhatme (1985) ${ }^{[8]}$. The values of $F_{1}$ averaged over replications were used for estimating heterosis. The magnitude of heterosis was calculated as percentage increase or decrease of $\mathrm{F}_{1}$ mean over the mean of better parent (BP) (Turner, 1953 and Hays et al. 1955) ${ }^{[13,2]}$. Similarly per cent superiority over the top parent (Top) and standard hybrid check (SC) were calculated.

\section{Results and Discussion}

For fifteen different characters the magnitude of heterosis noted in percentage increase or decrease over better parent, top parent and standard check which was presented in Table 1. Developed varieties which matures early in all seasons is the always desirable trait for realizing the highest economic yield as soon as possible in less time which is helpful and important ultimate goal for vegetable growers. As earliness is a desirable character in ridge gourd, the significantly negative heterosis is considered desirable for all maturity traits viz., length of internode, days to $50 \%$ flowering, length of pedicel and days required for first harvest. While positive heterosis considered desirable for rest of the other characters.

For length of vine, the cross combinations $2 \times 7$ and $3 \times 7$ displayed positive heterosis over better parent and standard check. The result was correspondence with Abusaleha and Dutta (1994) and Rao et al. (2000) ${ }^{[1,9]}$. While for length of internode, the crosses $1 \times 3$ and $3 \times 6$ expressed statistically highly significant negative heterosis over better parent, top parent and standard check. Number of nodes per vine, the cross combinations viz., $2 \times 7,3 \times 5$ and $3 \times 7$ reported highest and positive heterosis over better parent and $2 \times 7$ in standard check, respectively. Rao et al. (2000) and Mole et al. (2001) ${ }^{[9,5]}$ also reported significant positive heterosis for this traits.
For Days to $50 \%$ flowering, the cross combination only one 1 x 2 showed significantly negative heterosis over top parent. The crosses viz., $1 \times 3,5 \times 8,6 \times 7,6 \times 8$ and $7 \times 8$ showed significant negative heterosis over better parent and top parent for days required for first harvest. Early flowering was responsible for early fruiting reported by Karmakar (2011) and Narasannavar et al. (2014) ${ }^{[4,6]}$. For number of female flowers, the significant positive heterosis resulted in crosses 1 × $3,1 \times 5,1 \times 6,1 \times 8,2 \times 3,2 \times 5,2 \times 6,2 \times 7,3 \times 6,4 \times 6,4$ $\mathrm{x} 7$ and $6 \times 8$ over better parent, top parent and standard check. Similar findings supported by Niyaria and Bhalala (2001) and Shaha and Kale (2003) [7, 12]. The cross combinations $1 \times 2,1 \times 5,4 \times 7,4 \times 8$ and $5 \times 6$ resulted highest magnitude of significantly negative heterosis over better parent, top parent and standard check, for length of pedicel. The highest and positive heterosis exhibited by the cross combinations $1 \times 2,1 \times 4,1 \times 8,2 \times 5,2 \times 6,3 \times 6,3 \times$ $7,3 \times 8,4 \times 7,4 \times 8$ and $7 \times 8$ for length of fruit over better parent, top parent and standard check. For positive heterosis for length of fruit reported earlier by Rao and Rao (2002) and Hedau and Sirohi (2004) ${ }^{[10,3]}$. As regard to diameter of fruit, 3 crosses over better parent, only one cross over top parent and 4 crosses over standard check showed significantly positive heterosis. These results are in correspondence with those reported by Abusaleha and Dutta (1994) and Mole et al. (2001) [1, 5]. For number of fruits per vine, the cross combinations $1 \times 8,2 \times 7,2 \times 8,5 \times 8,6 \times 7,6 \times 8$ and $7 \times 8$ recorded highest positively significant heterosis over better parent, top parent and standard check. As regard the weight of fruit out of $28 \mathrm{~F}_{1}$ hybrids, 12 crosses over better parent and 12 crosses over top parent and 5 crosses over standard check revealed significant positive heterosis. Significantly positive heterosis were recorded in 14 crosses over better parent, 8 crosses over top parent and 11 crosses over standard check for weight of fruits per vine. Out of 28 crosses, 13 crosses over better parent, 8 crosses over top parent and 9 crosses over standard check indicated significantly positive heterosis for weight of fruits per plot and fruit yield $(\mathrm{q} / \mathrm{ha})$. In previous studies Hedau and Sirohi (2004) and Narasannavar et al. $(2014)^{[3,6]}$ also recorded significant positive heterosis for number of fruits per vine, weight of fruit, weight of fruits per vine and fruit yield ( $\mathrm{q} / \mathrm{ha})$.

Table 1: Heterosis (\%) over better parent, top parent and standard hybrid check in $8 \times 8$ half diallel in ridge gourd

\begin{tabular}{|c|c|c|c|c|c|c|c|}
\hline \multirow{2}{*}{ Sr. No. } & \multirow{2}{*}{ Crosses } & \multicolumn{3}{|c|}{ Length of vine $(\mathrm{cm})$} & \multicolumn{3}{|c|}{ Length of internode (cm) } \\
\hline & & B.P. & T.P. & S.C. & B.P. & T.P. & S.C. \\
\hline 1. & $1 \times 2$ & 11.82 & 0.53 & 2.78 & -5.56 & -9.91 & -1.45 \\
\hline 2. & $1 \times 3$ & -3.54 & -13.28 & -11.33 & $-15.17 *$ & $-20.45^{* *}$ & $-12.98 *$ \\
\hline 3. & $1 \times 4$ & -4.96 & -4.96 & -2.82 & 6.30 & -5.70 & 3.15 \\
\hline 4. & $1 \times 5$ & -7.98 & -11.50 & -9.52 & $-12.59 *$ & $-12.59 *$ & -4.38 \\
\hline 5. & $1 \times 6$ & 14.95 & 12.18 & 14.70 & 7.37 & 1.12 & 10.62 \\
\hline 6. & $1 \times 7$ & 21.43 & 9.17 & 11.63 & $16.81^{*}$ & -2.94 & 6.18 \\
\hline 7. & $1 \times 8$ & 20.11 & 8.73 & 11.17 & 1.19 & -6.77 & 1.98 \\
\hline 8. & $2 \times 3$ & -7.03 & -17.87 & -16.03 & $-14.10^{*}$ & $-18.06^{* *}$ & -10.37 \\
\hline 9. & $2 \times 4$ & 11.27 & 11.27 & 13.77 & 1.27 & -3.40 & 5.67 \\
\hline 10. & $2 \times 5$ & -8.42 & -11.93 & -9.95 & -6.11 & -6.11 & 2.71 \\
\hline 11. & $2 \times 6$ & 0.89 & -1.55 & 0.67 & -3.71 & -8.15 & 0.47 \\
\hline 12. & $2 \times 7$ & $36.70 * *$ & $20.27 *$ & 22.97* & 0.15 & -4.46 & 4.51 \\
\hline 13. & $2 \times 8$ & 17.84 & 6.67 & 9.06 & -2.02 & -6.54 & 2.24 \\
\hline 14. & $3 \times 4$ & 1.74 & 1.74 & 4.03 & -0.34 & -6.54 & 2.24 \\
\hline 15. & $3 \times 5$ & 12.30 & 7.99 & 10.42 & -7.40 & -7.40 & 1.29 \\
\hline 16. & $3 \times 6$ & -12.52 & -14.63 & -12.71 & $-19.63 * *$ & $-24.31 * *$ & $-17.20 * *$ \\
\hline 17. & $3 \times 7$ & $35.48 * *$ & 19.69 & $22.38 *$ & 3.38 & -3.05 & 6.05 \\
\hline 18. & $3 \times 8$ & 18.43 & 7.20 & 9.61 & 1.84 & -4.49 & 4.47 \\
\hline 19. & $4 \times 5$ & 15.96 & 15.96 & 18.56 & -3.37 & -3.37 & 5.70 \\
\hline 20. & $4 \times 6$ & -1.11 & -1.11 & 1.11 & -3.85 & -9.45 & -0.95 \\
\hline
\end{tabular}




\begin{tabular}{|l|c|c|c|c|c|c|c|}
\hline 21. & $4 \times 7$ & 7.24 & 7.24 & 9.65 & 5.88 & -6.08 & 2.74 \\
\hline 22. & $4 \times 8$ & 7.67 & 7.67 & 10.09 & 0.38 & -7.52 & 1.17 \\
\hline 23. & $5 \times 6$ & -8.36 & -10.57 & -8.56 & $-18.66^{* *}$ & $-18.66^{* *}$ & -11.03 \\
\hline 24. & $5 \times 7$ & -10.09 & -13.53 & -11.59 & $-16.85^{* *}$ & $-16.85^{* *}$ & -9.04 \\
\hline 25. & $5 \times 8$ & -1.14 & -4.92 & -2.79 & -10.80 & -10.80 & -2.43 \\
\hline 26. & $6 \times 7$ & 1.73 & -0.72 & 1.51 & -9.72 & $-14.98^{*}$ & -6.99 \\
\hline 27. & $6 \times 8$ & 18.01 & 15.16 & 17.75 & 3.46 & -2.56 & 6.58 \\
\hline 28. & $7 \times 8$ & 12.55 & 1.88 & 4.17 & -5.56 & $-12.99^{*}$ & -4.82 \\
\hline \multicolumn{2}{|c|}{ S.E. \pm C.D. 5\% } & 63.56 & 63.56 & 63.56 & 0.98 & 0.98 & 0.98 \\
\hline \multicolumn{2}{|c|}{ C.D. 1\% } & 129.04 & 129.04 & 129.04 & 2.00 & 2.00 & 2.00 \\
\hline
\end{tabular}

*and ** Significant at 5\% and $1 \%$ level

\begin{tabular}{|c|c|c|c|c|c|c|c|}
\hline \multirow{2}{*}{ Sr. No. } & \multirow{2}{*}{ Crosses } & \multicolumn{3}{|c|}{ Number of nodes per vine } & \multicolumn{3}{|c|}{ Days to $50 \%$ flowering } \\
\hline & & B.P. & T.P. & S.C. & B.P. & T.P. & S.C. \\
\hline 1. & $1 \times 2$ & 2.23 & -0.96 & 4.04 & -14.74 & $-19.08 *$ & -11.74 \\
\hline 2. & $1 \times 3$ & -0.25 & -3.37 & 1.52 & -1.06 & -8.41 & -0.11 \\
\hline 3. & $1 \times 4$ & -10.58 & -10.58 & -6.06 & 6.15 & -0.29 & 8.75 \\
\hline 4. & $1 \times 5$ & -7.20 & -10.10 & -5.56 & -13.60 & -13.60 & -5.76 \\
\hline 5. & $1 \times 6$ & 1.74 & -1.44 & 3.54 & -4.66 & -5.87 & 2.67 \\
\hline 6. & $1 \times 7$ & 2.98 & -0.24 & 4.80 & 1.46 & -4.79 & 3.84 \\
\hline 7. & $1 \times 8$ & 6.70 & 3.37 & 8.59 & -7.88 & -10.76 & -2.67 \\
\hline 8. & $2 \times 3$ & 6.32 & -11.06 & -6.57 & -4.02 & -8.90 & -0.64 \\
\hline 9. & $2 \times 4$ & 1.92 & 1.92 & 7.07 & 10.72 & 5.09 & 14.62 \\
\hline 10. & $2 \times 5$ & -2.54 & $-16.83^{*}$ & -12.63 & -9.00 & -9.00 & -0.75 \\
\hline 11. & $2 \times 6$ & 3.39 & -4.81 & 0.00 & -5.25 & -6.46 & 2.03 \\
\hline 12. & $2 \times 7$ & $18.97 *$ & 11.54 & $17.17 *$ & 0.41 & -4.70 & 3.95 \\
\hline 13. & $2 \times 8$ & 15.34 & 1.20 & 6.31 & 6.97 & 3.62 & 13.02 \\
\hline 14. & $3 \times 4$ & -3.37 & -3.37 & 1.52 & -8.23 & -13.80 & -5.98 \\
\hline 15. & $3 \times 5$ & $21.13 *$ & 3.37 & 8.59 & -12.23 & -12.23 & -4.27 \\
\hline 16. & $3 \times 6$ & 8.62 & 0.00 & 5.05 & -5.75 & -6.95 & 1.49 \\
\hline 17. & $3 \times 7$ & $16.67 *$ & 9.38 & 14.90 & -2.71 & -8.71 & -0.43 \\
\hline 18. & $3 \times 8$ & 13.42 & -0.48 & 4.55 & -1.11 & -4.21 & 4.48 \\
\hline 19. & $4 \times 5$ & 6.25 & 6.25 & 11.62 & -2.84 & -2.84 & 5.98 \\
\hline 20. & $4 \times 6$ & -3.12 & -3.12 & 1.77 & 0.79 & -0.49 & 8.54 \\
\hline 21. & $4 \times 7$ & 1.20 & 1.20 & 6.31 & 0.00 & -6.07 & 2.45 \\
\hline 22. & $4 \times 8$ & 3.37 & 3.37 & 8.59 & 7.37 & 4.01 & 13.45 \\
\hline 23. & $5 \times 6$ & 6.01 & -2.40 & 2.53 & 1.96 & 1.96 & 11.21 \\
\hline 24. & $5 \times 7$ & -1.54 & -7.69 & -3.03 & -7.93 & -7.93 & 0.43 \\
\hline 25. & $5 \times 8$ & 7.67 & -5.53 & -0.76 & -11.94 & -11.94 & -3.95 \\
\hline 26. & $6 \times 7$ & 11.03 & 4.09 & 9.34 & -13.88 & -14.97 & -7.26 \\
\hline 27. & $6 \times 8$ & 14.36 & 5.29 & 10.61 & -10.41 & -11.55 & -3.52 \\
\hline 28. & $7 \times 8$ & 10.26 & 3.37 & 8.59 & -4.95 & -7.93 & 0.43 \\
\hline \multicolumn{2}{|c|}{ S.E. \pm} & 2.96 & 2.96 & 2.96 & 3.88 & 3.88 & 3.88 \\
\hline \multicolumn{2}{|c|}{ C.D. $5 \%$} & 6.02 & 6.02 & 6.02 & 7.89 & 7.89 & 7.89 \\
\hline \multicolumn{2}{|c|}{ C.D. $1 \%$} & 8.08 & 8.08 & 8.08 & 10.59 & 10.59 & 10.59 \\
\hline
\end{tabular}

*and ** Significant at $5 \%$ and $1 \%$ level

\begin{tabular}{|c|c|c|c|c|c|c|c|}
\hline \multirow{2}{*}{ Sr. No. } & \multirow{2}{*}{ Crosses } & \multicolumn{3}{|c|}{ Days required for first harvest } & \multicolumn{3}{|c|}{ No. of female flowers } \\
\cline { 2 - 7 } & B.P. & T.P. & S.C. & B.P. & \multicolumn{2}{c|}{ T.C. } \\
\hline 1. & $1 \times 2$ & $-11.17^{* *}$ & $-16.13^{* *}$ & -0.94 & -13.64 & $-18.43^{*}$ & -9.52 \\
\hline 2. & $1 \times 3$ & $-14.45^{* *}$ & $-16.77^{* *}$ & -1.70 & $-32.09^{* *}$ & $-35.86^{* *}$ & $-28.85^{* *}$ \\
\hline 3. & $1 \times 4$ & -4.23 & $-9.58^{*}$ & 6.79 & -11.36 & -11.36 & -1.68 \\
\hline 4. & $1 \times 5$ & $-8.80^{*}$ & $-13.90^{* *}$ & 1.70 & $-38.24^{* *}$ & $-41.67^{* *}$ & $-35.29^{* *}$ \\
\hline 5. & $1 \times 6$ & $-12.56^{* *}$ & $-14.38^{* *}$ & 1.13 & $-28.34^{* *}$ & $-32.32^{* *}$ & $-24.93^{*}$ \\
\hline 6. & $1 \times 7$ & $-8.15^{*}$ & $-8.15^{*}$ & 8.49 & -4.81 & -10.10 & -0.28 \\
\hline 7. & $1 \times 8$ & $-11.45^{* *}$ & $-14.54^{* *}$ & 0.94 & $37.97^{* *}$ & $30.30^{* *}$ & $44.54^{* *}$ \\
\hline 8. & $2 \times 3$ & -7.22 & $-9.74^{*}$ & 6.60 & $-27.54^{* *}$ & $-36.87^{* *}$ & $-29.97^{* *}$ \\
\hline 9. & $2 \times 4$ & -2.62 & $-10.86^{* *}$ & 5.28 & -11.36 & -11.36 & -1.68 \\
\hline 10. & $2 \times 5$ & -1.95 & $-11.82^{* *}$ & 4.15 & $-35.65^{* *}$ & $-43.94^{* *}$ & $-37.82^{* *}$ \\
\hline 11. & $2 \times 6$ & $-9.30^{*}$ & $-11.18^{* *}$ & 4.91 & $-22.99^{*}$ & $-34.85^{* *}$ & $-27.73^{* *}$ \\
\hline 12. & $2 \times 7$ & $-10.86^{* *}$ & $-10.86^{* *}$ & 5.28 & $42.69^{* *}$ & $20.71^{*}$ & $33.89^{* *}$ \\
\hline 13. & $2 \times 8$ & -6.29 & $-9.58^{*}$ & 6.79 & 17.38 & 4.04 & 15.41 \\
\hline 14. & $3 \times 4$ & $-12.32^{* *}$ & $-14.70^{* *}$ & 0.75 & -6.06 & -6.06 & 4.20 \\
\hline 15. & $3 \times 5$ & $-10.34^{*}$ & $-12.78^{* *}$ & 3.02 & 13.33 & -1.26 & 9.52 \\
\hline 16. & $3 \times 6$ & $-14.19^{* *}$ & $-15.97^{* *}$ & -0.75 & $-26.09^{*}$ & $-35.61^{* *}$ & $-28.57^{* *}$ \\
\hline 17. & $3 \times 7$ & $-11.34^{* *}$ & $-11.34^{* *}$ & 4.72 & -5.51 & -17.68 & -8.68 \\
\hline 18. & $3 \times 8$ & $-14.29^{* *}$ & $-16.61^{* *}$ & -1.51 & -5.98 & -16.67 & -7.56 \\
\hline 19. & $4 \times 5$ & 0.00 & $-8.47^{*}$ & 8.11 & -15.40 & -15.40 & -6.16 \\
\hline
\end{tabular}




\begin{tabular}{|c|c|c|c|c|c|c|c|}
\hline 20. & $4 \times 6$ & -6.20 & $-8.15^{*}$ & 8.49 & $-28.03 * *$ & $-28.03 * *$ & $-20.17 *$ \\
\hline 21. & $4 \times 7$ & -4.79 & -4.79 & $12.45 * *$ & $-28.54 * *$ & $-28.54 * *$ & $-20.73 *$ \\
\hline 22. & $4 \times 8$ & -1.49 & -4.95 & $12.26 * *$ & -13.64 & -13.64 & -4.20 \\
\hline 23. & $5 \times 6$ & -3.75 & -5.75 & $11.32 *$ & 4.06 & -9.34 & 0.56 \\
\hline 24. & $5 \times 7$ & $-10.06^{*}$ & $-10.06^{*}$ & 6.23 & 10.72 & -3.54 & 7.00 \\
\hline 25. & $5 \times 8$ & $-16.39 * *$ & $-19.33 * *$ & -4.72 & $21.94 *$ & 8.08 & $19.89 *$ \\
\hline 26. & $6 \times 7$ & $-19.33 * *$ & $-19.33^{* *}$ & -4.72 & $40.88 * *$ & 5.30 & 16.81 \\
\hline 27. & $6 \times 8$ & $-19.25 * *$ & $-20.93 * *$ & -6.60 & $49.00 * *$ & $32.07 * *$ & $46.50 * *$ \\
\hline 28. & $7 \times 8$ & $-21.73 * *$ & $-21.73 * *$ & -7.55 & 18.80 & 5.30 & 16.81 \\
\hline \multicolumn{2}{|c|}{ S.E. \pm} & 2.38 & 2.38 & 2.38 & 3.47 & 3.47 & 3.47 \\
\hline \multicolumn{2}{|c|}{ C.D. 5\% } & 4.83 & 4.83 & 4.83 & 7.04 & 7.04 & 7.04 \\
\hline \multicolumn{2}{|c|}{ C.D. $1 \%$} & 6.48 & 6.48 & 6.48 & 9.45 & 9.45 & 9.45 \\
\hline
\end{tabular}

*and ** Significant at $5 \%$ and $1 \%$ level

\begin{tabular}{|c|c|c|c|c|c|c|c|}
\hline \multirow{2}{*}{ Sr. No. } & \multirow{2}{*}{ Crosses } & \multicolumn{3}{|c|}{ Length of pedicel (cm) } & \multicolumn{3}{|c|}{ Length of fruit $(\mathrm{cm})$} \\
\hline & & B.P. & T.P. & S.C. & B.P. & T.P. & S.C. \\
\hline 1. & $1 \times 2$ & $-21.01 * *$ & $-21.01 * *$ & $-38.26 * *$ & $27.27 * *$ & $11.53^{*}$ & $12.67 *$ \\
\hline 2. & $1 \times 3$ & $13.19 * *$ & $13.19 * *$ & $-11.53 * *$ & $23.03 * *$ & 7.81 & 8.91 \\
\hline 3. & $1 \times 4$ & $11.46 * *$ & $11.46^{* *}$ & $-12.89 * *$ & $27.71 * *$ & $11.92 *$ & $13.06^{*}$ \\
\hline 4. & $1 \times 5$ & $-9.37 *$ & $-9.37 *$ & $-29.17 * *$ & -11.27 & $-19.20 * *$ & $-18.37 * *$ \\
\hline 5. & $1 \times 6$ & $-25.00 * *$ & $-25.00 * *$ & $-41.38 * *$ & $17.34 * *$ & 2.83 & 3.88 \\
\hline 6. & $1 \times 7$ & $17.01 * *$ & $17.01 * *$ & $-8.55^{*}$ & $14.57 *$ & 0.40 & 1.43 \\
\hline 7. & $1 \times 8$ & -3.47 & -3.47 & $-24.56 * *$ & $17.70 * *$ & $17.70 * *$ & $18.91 * *$ \\
\hline 8. & $2 \times 3$ & $29.81 * *$ & $17.19 * *$ & $-8.41 *$ & $19.00 * *$ & 2.19 & 3.24 \\
\hline 9. & $2 \times 4$ & $30.59 * *$ & $26.74 * *$ & -0.95 & 8.49 & -8.40 & -7.47 \\
\hline 10. & $2 \times 5$ & 1.08 & -2.78 & $-24.02 * *$ & $22.04 * *$ & $11.14^{*}$ & $12.27 *$ \\
\hline 11. & $2 \times 6$ & $47.12 * *$ & $32.81 * *$ & 3.80 & $59.98 * *$ & $19.66 * *$ & $20.89 * *$ \\
\hline 12. & $2 \times 7$ & $-25.80 * *$ & $-27.08 * *$ & $-43.01 * *$ & 6.23 & $-13.32 *$ & $-12.43^{*}$ \\
\hline 13. & $2 \times 8$ & 0.00 & -5.21 & $-25.92 * *$ & $-13.97 *$ & $-13.97 *$ & $-13.09 *$ \\
\hline 14. & $3 \times 4$ & $-8.77 *$ & $-11.46^{* *}$ & $-30.80 * *$ & $29.49 * *$ & $11.20^{*}$ & $12.34 *$ \\
\hline 15. & $3 \times 5$ & $16.79 * *$ & $12.33 * *$ & $-12.21 * *$ & 7.65 & -1.96 & -0.96 \\
\hline 16. & $3 \times 6$ & $11.54 *$ & $-9.37 *$ & $-29.17 * *$ & $30.94 * *$ & $12.45^{*}$ & $13.59 *$ \\
\hline 17. & $3 \times 7$ & $13.60 * *$ & $11.63 * *$ & $-12.75^{* *}$ & $31.45^{* *}$ & $12.88^{*}$ & $14.03^{*}$ \\
\hline 18. & $3 \times 8$ & $15.20 * *$ & $9.20 *$ & $-14.65 * *$ & $13.44 *$ & 13.44* & $14.60^{*}$ \\
\hline 19. & $4 \times 5$ & 1.07 & -1.91 & $-23.34 * *$ & 8.03 & -1.62 & -0.61 \\
\hline 20. & $4 \times 6$ & $15.38 * *$ & $11.98 * *$ & $-12.48 * *$ & $30.15 * *$ & 9.88 & 11.00 \\
\hline 21. & $4 \times 7$ & $-18.02 * *$ & $-19.44 * *$ & $-37.04 * *$ & $32.01 * *$ & $11.45^{*}$ & $12.59 *$ \\
\hline 22. & $4 \times 8$ & -6.26 & $-9.03 *$ & $-28.90 * *$ & $29.06 * *$ & $29.06 * *$ & $30.38 * *$ \\
\hline 23. & $5 \times 6$ & -5.23 & $-8.85^{*}$ & $-28.77 * *$ & 0.22 & -8.73 & -7.80 \\
\hline 24. & $5 \times 7$ & -0.71 & -2.43 & $-23.74 * *$ & 0.34 & -8.62 & -7.69 \\
\hline 25. & $5 \times 8$ & -2.35 & -6.08 & $-26.59 * *$ & -3.42 & -3.42 & -2.44 \\
\hline 26. & $6 \times 7$ & $-16.25 * *$ & $-17.71 * *$ & $-35.69 * *$ & $22.59 * *$ & 0.03 & 1.05 \\
\hline 27. & $6 \times 8$ & -2.01 & -7.12 & $-27.41 * *$ & -0.50 & -0.50 & 0.52 \\
\hline 28. & $7 \times 8$ & $35.16 * *$ & $32.81 * *$ & 3.80 & $13.81^{*}$ & $13.81^{*}$ & $14.98^{*}$ \\
\hline \multicolumn{2}{|c|}{ S.E. \pm} & 0.23 & 0.23 & 0.23 & 1.75 & 1.75 & 1.75 \\
\hline \multicolumn{2}{|c|}{ C.D. $5 \%$} & 0.48 & 0.48 & 0.48 & 3.56 & 3.56 & 3.56 \\
\hline \multicolumn{2}{|c|}{ C.D. $1 \%$} & 0.65 & 0.65 & 0.65 & 4.78 & 4.78 & 4.78 \\
\hline
\end{tabular}

$*$ and $* *$ Significant at $5 \%$ and $1 \%$ level

\begin{tabular}{|c|c|c|c|c|c|c|c|}
\hline \multirow{2}{*}{ Sr. No. } & \multirow{2}{*}{ Crosses } & \multicolumn{3}{|c|}{ Diameter of fruit (cm) } & \multicolumn{3}{|c|}{ No. of fruits per vine } \\
\hline & & B.P. & T.P. & S.C. & B.P. & T.P. & S.C. \\
\hline 1. & $1 \times 2$ & 4.19 & -0.57 & $23.19^{*}$ & -15.24 & $-24.17 *$ & -9.74 \\
\hline 2. & $1 \times 3$ & 9.43 & 4.43 & $29.38 * *$ & -14.52 & $-31.10 * *$ & -17.99 \\
\hline 3. & $1 \times 4$ & $-15.14 *$ & $-15.14 *$ & 5.13 & $-22.41 *$ & $-32.27 * *$ & -19.38 \\
\hline 4. & $1 \times 5$ & -8.98 & -13.14 & 7.61 & $-29.95 *$ & $-41.82 * *$ & $-30.75^{*}$ \\
\hline 5. & $1 \times 6$ & -5.84 & -10.14 & 11.33 & $-22.73 *$ & $-34.53 * *$ & -22.08 \\
\hline 6. & $1 \times 7$ & -12.57 & $-16.57 *$ & 3.36 & 8.29 & -12.71 & 3.90 \\
\hline 7. & $1 \times 8$ & -4.19 & -8.57 & 13.27 & $66.94 * *$ & $66.94 * *$ & $98.70^{* * *}$ \\
\hline 8. & $2 \times 3$ & 14.44 & 3.00 & $27.61 * *$ & $-44.51 * *$ & $-50.35^{* *}$ & $-40.91 * *$ \\
\hline 9. & $2 \times 4$ & 4.29 & 4.29 & $29.20 * *$ & -1.83 & -12.17 & 4.55 \\
\hline 10. & $2 \times 5$ & 9.84 & -1.14 & $22.18 *$ & $-42.38 * *$ & $-48.45^{* *}$ & $-38.64 * *$ \\
\hline 11. & $2 \times 6$ & -6.75 & -13.14 & 7.61 & $-26.83 *$ & $-34.53 * *$ & -22.08 \\
\hline 12. & $2 \times 7$ & -3.17 & -12.86 & 7.96 & $62.35 * *$ & $45.25 * *$ & $72.89 * *$ \\
\hline 13. & $2 \times 8$ & 15.08 & 3.57 & $28.32 * *$ & $41.38 * *$ & $41.38 * *$ & $68.28 * *$ \\
\hline 14. & $3 \times 4$ & $-24.71 * *$ & $-24.71 * *$ & -6.73 & 4.69 & -8.62 & 8.77 \\
\hline 15. & $3 \times 5$ & 8.72 & -5.57 & 16.99 & 14.65 & -4.77 & 13.34 \\
\hline 16. & $3 \times 6$ & -3.83 & -10.43 & 10.97 & $-33.03 * *$ & $-43.26^{* *}$ & $-32.47 * *$ \\
\hline 17. & $3 \times 7$ & 10.71 & -2.57 & $20.71 *$ & $57.16 * *$ & -6.55 & 11.23 \\
\hline 18. & $3 \times 8$ & -2.42 & -13.43 & 7.26 & -17.62 & -17.62 & -1.95 \\
\hline
\end{tabular}




\begin{tabular}{|c|c|c|c|c|c|c|c|}
\hline 19. & $4 \times 5$ & -12.71 & -12.71 & 8.14 & 12.75 & -1.58 & 17.14 \\
\hline 20. & $4 \times 6$ & -12.71 & -12.71 & 8.14 & $-25.63^{*}$ & $-35.08^{* *}$ & -22.73 \\
\hline 21. & $4 \times 7$ & 5.86 & 5.86 & $31.15^{* *}$ & $-28.13^{*}$ & $-37.26^{* *}$ & $-25.32^{*}$ \\
\hline 22. & $4 \times 8$ & 0.43 & 0.43 & $24.42^{* *}$ & 9.55 & 9.55 & $30.39^{*}$ \\
\hline 23. & $5 \times 6$ & -8.74 & $-15.00^{*}$ & 5.31 & 18.32 & 0.25 & 19.32 \\
\hline 24. & $5 \times 7$ & $20.13^{*}$ & 5.71 & $30.97^{* *}$ & 17.27 & -2.59 & 15.94 \\
\hline 25. & $5 \times 8$ & 5.80 & -6.14 & 16.28 & $40.21^{* *}$ & $40.21^{* *}$ & $66.88^{* *}$ \\
\hline 26. & $6 \times 7$ & -10.89 & $-17.00^{*}$ & 2.83 & $65.49^{* *}$ & $40.21^{* *}$ & $66.88^{* *}$ \\
\hline 27. & $6 \times 8$ & -7.98 & -14.29 & 6.19 & $72.94^{* *}$ & $72.94^{* *}$ & $105.84^{* *}$ \\
\hline 28. & $7 \times 8$ & -5.31 & $-16.00^{*}$ & 4.07 & $21.11^{*}$ & $21.11^{*}$ & $44.16^{* *}$ \\
\hline \multicolumn{2}{|c|}{ S.E. \pm} & 0.25 & 0.25 & 0.25 & 1.77 & 1.77 & 1.77 \\
\hline \multicolumn{2}{|c|}{ C.D. 5\% } & 0.51 & 0.51 & 0.51 & 3.59 & 3.59 & 3.59 \\
\hline \multicolumn{2}{|c|}{ C.D. $1 \%$} & 0.68 & 0.68 & 0.68 & 4.82 & 4.82 & 4.82 \\
\hline
\end{tabular}

*and ** Significant at 5\% and $1 \%$ level

\begin{tabular}{|c|c|c|c|c|c|c|c|}
\hline \multirow{2}{*}{ Sr. No. } & \multirow{2}{*}{ Crosses } & \multicolumn{3}{|c|}{ Weight of fruit (g) } & \multicolumn{3}{|c|}{ Weight of fruits per vine (kg) } \\
\hline & & B.P. & T.P. & S.C. & B.P. & T.P. & S.C. \\
\hline 1. & $1 \times 2$ & $21.42 * *$ & $17.83 * *$ & $12.62^{*}$ & 8.48 & -1.38 & 6.55 \\
\hline 2. & $1 \times 3$ & $25.02 * *$ & $23.03 * *$ & $17.59 * *$ & -3.73 & $-21.76^{*}$ & -15.48 \\
\hline 3. & $1 \times 4$ & $19.97 * *$ & $15.43^{* *}$ & 10.32 & 0.63 & -11.29 & -4.17 \\
\hline 4. & $1 \times 5$ & 5.05 & 0.89 & -3.57 & $-25.58^{*}$ & $-38.29 * *$ & $-33.33 * *$ \\
\hline 5. & $1 \times 6$ & $17.72 * *$ & $13.44 *$ & 8.42 & -9.03 & $-22.31 *$ & -16.07 \\
\hline 6. & $1 \times 7$ & $11.90 *$ & $11.90^{*}$ & 6.95 & $36.95 * *$ & 11.29 & 20.24 \\
\hline 7. & $1 \times 8$ & 8.06 & 3.78 & -0.81 & $82.09 * *$ & $82.09 * *$ & $96.73 * *$ \\
\hline 8. & $2 \times 3$ & -0.61 & -2.20 & -6.52 & $-43.94 * *$ & $-49.04 * *$ & $-44.94 * *$ \\
\hline 9. & $2 \times 4$ & $22.91 * *$ & $19.28 * *$ & $14.01 *$ & 20.91 & 9.92 & 18.75 \\
\hline 10. & $2 \times 5$ & -8.25 & -10.96 & $-14.90 * *$ & $-46.97 * *$ & $-51.79 * *$ & $-47.92 * *$ \\
\hline 11. & $2 \times 6$ & -2.42 & -5.30 & -9.49 & $-27.58^{*}$ & $-34.16 * *$ & $-28.87 *$ \\
\hline 12. & $2 \times 7$ & 6.01 & 6.01 & 1.33 & $77.58 * *$ & $61.43^{* *}$ & $74.40 * *$ \\
\hline 13. & $2 \times 8$ & $14.80^{*}$ & $11.41^{*}$ & 6.48 & $64.74 * *$ & $64.74 * *$ & $77.98 * *$ \\
\hline 14. & $3 \times 4$ & $18.41 * *$ & $16.52 * *$ & $11.37 *$ & $26.88 *$ & 11.85 & 20.83 \\
\hline 15. & $3 \times 5$ & $17.32 * *$ & $15.45 * *$ & 10.34 & $38.87 * *$ & 15.15 & $24.40^{*}$ \\
\hline 16. & $3 \times 6$ & 7.23 & 5.52 & 0.85 & $-26.13^{*}$ & $-36.91 * *$ & $-31.85^{* *}$ \\
\hline 17. & $3 \times 7$ & 1.21 & 1.21 & -3.27 & $81.78 * *$ & 12.67 & 21.73 \\
\hline 18. & $3 \times 8$ & 11.06 & 9.29 & 4.46 & -4.68 & -4.68 & 2.98 \\
\hline 19. & $4 \times 5$ & -1.24 & -4.98 & -9.18 & 11.25 & -1.93 & 5.95 \\
\hline 20. & $4 \times 6$ & $18.65 * *$ & $14.33^{*}$ & 9.28 & -11.25 & $-21.76^{*}$ & -15.48 \\
\hline 21. & $4 \times 7$ & 4.39 & 4.39 & -0.23 & -20.63 & $-30.03 * *$ & $-24.40 *$ \\
\hline 22. & $4 \times 8$ & $19.66 * *$ & $15.14^{*}$ & 10.04 & $32.23 * *$ & $32.23 * *$ & $42.86 * *$ \\
\hline 23. & $5 \times 6$ & 6.97 & 3.09 & -1.47 & $38.71 * *$ & 18.46 & $27.98^{*}$ \\
\hline 24. & $5 \times 7$ & 11.36 & 11.36 & 6.44 & $43.19 * *$ & 18.73 & $28.27 *$ \\
\hline 25. & $5 \times 8$ & $23.06^{* *}$ & $17.32 * *$ & $12.14^{*}$ & $72.73 * *$ & $72.73 * *$ & $86.61 * *$ \\
\hline 26. & $6 \times 7$ & 3.05 & 3.05 & -1.50 & $75.81 * *$ & $50.14 * *$ & $62.20 * *$ \\
\hline 27. & $6 \times 8$ & -3.22 & -6.73 & -10.86 & $77.41 * *$ & $77.41 * *$ & $91.67 * *$ \\
\hline 28. & $7 \times 8$ & 5.90 & 5.90 & 1.22 & $34.44 * *$ & $34.44 * *$ & $45.24 * *$ \\
\hline \multicolumn{2}{|c|}{ S.E. \pm} & 5.83 & 5.83 & 5.83 & 0.18 & 0.18 & 0.18 \\
\hline \multicolumn{2}{|c|}{ C.D. $5 \%$} & 11.83 & 11.83 & 11.83 & 0.37 & 0.37 & 0.37 \\
\hline \multicolumn{2}{|c|}{ C.D. $1 \%$} & 15.88 & 15.88 & 15.88 & 0.50 & 0.50 & 0.50 \\
\hline
\end{tabular}

*and ** Significant at $5 \%$ and $1 \%$ level

\begin{tabular}{|c|c|c|c|c|c|c|c|}
\hline \multirow{2}{*}{ Sr. No. } & \multirow{2}{*}{ Crosses } & \multicolumn{3}{|c|}{ Weight of fruits per plot (kg) } & \multicolumn{3}{c|}{ Fruit yield (q/ha) } \\
\cline { 3 - 7 } & & B.P. & T.P. & S.C. & B.P. & T.P. & S.C. \\
\hline 1. & $1 \times 2$ & 3.08 & -6.16 & 1.61 & 3.09 & -6.17 & 1.64 \\
\hline 2. & $1 \times 3$ & 10.05 & -10.84 & -3.46 & 10.04 & -10.90 & -3.49 \\
\hline 3. & $1 \times 4$ & -6.37 & -17.45 & -10.61 & -6.34 & -17.45 & -10.58 \\
\hline 4. & $1 \times 5$ & -25.42 & $-38.30^{* *}$ & $-33.19^{* *}$ & -25.38 & $-38.30^{* *}$ & $-33.17^{* *}$ \\
\hline 5. & $1 \times 6$ & -9.00 & -22.12 & -15.67 & -9.00 & -22.15 & -15.67 \\
\hline 6. & $1 \times 7$ & 26.63 & 2.59 & 11.08 & 26.65 & 2.55 & 11.09 \\
\hline 7. & $1 \times 8$ & $82.17^{* *}$ & $82.17^{* *}$ & $97.26^{* *}$ & $81.74^{* *}$ & $81.74^{* *}$ & $96.87^{* *}$ \\
\hline 8. & $2 \times 3$ & $-43.71^{* *}$ & $-48.76^{* *}$ & $-44.52^{* *}$ & $-44.12^{* *}$ & $-49.14^{* *}$ & $-44.90^{* *}$ \\
\hline 9. & $2 \times 4$ & 20.86 & 10.02 & 19.13 & 20.84 & 9.98 & 19.14 \\
\hline 10. & $2 \times 5$ & $-47.28^{* *}$ & $-52.01^{* *}$ & $-48.03^{* *}$ & $-47.27^{* *}$ & $-52.00^{* *}$ & $-48.01^{* *}$ \\
\hline 11. & $2 \times 6$ & $-27.99^{*}$ & $-34.45^{* *}$ & $-29.02^{*}$ & $-28.00^{*}$ & $-34.47^{* *}$ & $-29.01^{*}$ \\
\hline 12. & $2 \times 7$ & $77.27^{* *}$ & $61.36^{* *}$ & $74.73^{* *}$ & $77.14^{* *}$ & $61.22^{* *}$ & $74.64^{* *}$ \\
\hline 13. & $2 \times 8$ & $64.67^{* *}$ & $64.67^{* *}$ & $78.31^{* *}$ & $64.63^{* *}$ & $64.63^{* *}$ & $78.33^{* *}$ \\
\hline 14. & $3 \times 4$ & $26.90^{*}$ & 11.89 & 21.16 & $26.92^{*}$ & 11.87 & 21.18 \\
\hline 15. & $3 \times 5$ & $39.39^{* *}$ & 15.30 & $24.85^{*}$ & $39.43^{* *}$ & 15.29 & $24.88^{*}$ \\
\hline 16. & $3 \times 6$ & -26.17 & $-36.82^{* *}$ & $-31.59^{*}$ & -26.17 & $-36.83^{* *}$ & $-31.58^{*}$ \\
\hline 17. & $3 \times 7$ & $61.20^{* *}$ & -0.55 & 7.69 & $61.09^{* *}$ & -0.57 & 7.71 \\
\hline
\end{tabular}




\begin{tabular}{|c|c|c|c|c|c|c|c|}
\hline 18. & $3 \times 8$ & -4.51 & -4.51 & 3.40 & -4.52 & -4.52 & 3.42 \\
\hline 19. & $4 \times 5$ & 11.17 & -1.98 & 6.14 & 11.18 & -2.00 & 6.16 \\
\hline 20. & $4 \times 6$ & -11.24 & -21.74 & -15.26 & -11.19 & -21.72 & -15.20 \\
\hline 21. & $4 \times 7$ & -22.16 & $-31.37 * *$ & $-25.69 *$ & -22.08 & $-31.32 * *$ & $-25.60 *$ \\
\hline 22. & $4 \times 8$ & $32.09 * *$ & $32.09 * *$ & $43.03 * *$ & $32.01 * *$ & $32.01 * *$ & $43.00 * *$ \\
\hline 23. & $5 \times 6$ & $26.82 *$ & 8.53 & 17.52 & $26.79 *$ & 8.48 & 17.51 \\
\hline 24. & $5 \times 7$ & $37.92 * *$ & 14.09 & 23.54 & $37.98 * *$ & 14.09 & 23.59 \\
\hline 25. & $5 \times 8$ & $72.48 * *$ & $72.48 * *$ & $86.77 * *$ & $72.37 * *$ & $72.37 * *$ & $86.72 * *$ \\
\hline 26. & $6 \times 7$ & $77.17 * *$ & $51.62 * *$ & $64.18 * *$ & $77.20 * *$ & $51.60 * *$ & $64.22 * *$ \\
\hline 27. & $6 \times 8$ & $69.51 * *$ & $69.51 * *$ & $83.55 * *$ & $69.39 * *$ & $69.39 * *$ & $83.49 * *$ \\
\hline 28. & $7 \times 8$ & $34.40 * *$ & $34.40 * *$ & $45.53 * *$ & $34.31 * *$ & $34.31 * *$ & $45.49 * *$ \\
\hline \multicolumn{2}{|c|}{ S.E. \pm} & 1.01 & 1.01 & 1.01 & 13.54 & 13.54 & 13.54 \\
\hline \multicolumn{2}{|c|}{ C.D. $5 \%$} & 2.06 & 2.06 & 2.06 & 27.48 & 27.48 & 27.48 \\
\hline \multicolumn{2}{|c|}{ C.D. $1 \%$} & 2.76 & 2.76 & 2.76 & 36.88 & 36.88 & 36.88 \\
\hline
\end{tabular}

*and $* *$ Significant at $5 \%$ and $1 \%$ level

\section{Acknowledgement}

The authors thankful to the Department of Horticulture, PGI, Mahatma Phule Krishi Vidyapeeth, Rahuri (Maharashtra) for providing all the inputs and facilities to carry out this study.

\section{References}

1. Abusaleha T, Dutta OP. Manifestation of heterosis in ridge gourd [Luffa acutangula (L.) Roxb.]. Indian J Hort 1994;51(4):389-392.

2. Hays HR, Immer FR, Smith DC. Methods of plant Breeding, $2^{\text {nd }}$ edition, McGraw Hill Book publishing Company, Inc., New Delhi, 1955.

3. Hedau NK, Sirohi PS. Combining ability for yield and its contributing characters in ridge gourd. Prog. Hort 2004;36(1):91-93.

4. Karmakar P. Studies on heterosis and inheritance of hermaphorditism in ridge gourd (Luffa acutangula (L.) Roxb.) Ph.D. Thesis. IARI, New Delhi, 2011.

5. Mole TJ, Devi SN, Rajan S, Sadhan Kumar PG. Heterosis and combining ability in ridge gourd (Luffa acutangula (L.) Roxb.). Veg. Sci 2001;28(2):165-167.

6. Narasannavar AR, Gasti VD, Shantappa T, Mulge R, Allolli TB, Thammaiah N. Heterosis studies in ridge gourd (Luffa acutangula (L.) Roxb.). Karnataka J Agric Sci 2014;27(1):47-51.

7. Niyaria R, Bhalala MK. Heterosis and combining ability in ridge gourd. Indian $\mathrm{J} \mathrm{Pl}$. Genet. Resources 2001;14:101-102.

8. Panse VG, Sukhatme PV. Statistical Methods for Agricultural Workers, $4^{\text {th }}$ Edn., ICAR, New Delhi, 1985.

9. Rao BN, Rao PV, Reddy BM. Combining ability studies in ridge gourd (Luffa acutangula (L.) Roxb.). Int. J Trop Agri 2000;18(2):141-146.

10. Rao BN, Rao PV. Heterosis in ridge gourd. (Luffa acutangula (L.) Roxb.). J Res Angrau 2002;30(1):11-18.

11. Richharia RH. Intervarietal hybridization in Luffa acutangula and its economic significance. Proc. Bihar Acd. Agr. Sci 1952;1:1-9.

12. Shaha SR, Kale PN. Diallel analysis for combining ability in ridge gourd. J Maharashtra Agril Univ 2003;28(3):252-254.

13. Turner JH. A study of heterosis in upland cotton-I. yield of hybrid compared with varieties. Combining ability and inbreeding effects. Agronomy Journal 1953; 43:487-490. 